\title{
Chemical Transformation of Astaxanthin from Haematococcus pluvialis Improves its Antioxidative and Anti-inflammatory Activities
}

\author{
Sung Hyun Hwang ${ }^{1}$, Ji Min Kim¹, Seokjoon Kim¹, Min Jin Yoon ${ }^{2}$, Ki Soo Park ${ }^{1, *}$
}

${ }^{1}$ Department of Biological Engineering, College of Engineering, Konkuk University, Seoul 05029, Republic of Korea.

${ }^{2}$ Natural Bio Tree, Seongnam-si, Gyeonggi 13449, Republic of Korea.

*Correspondence: E-mail: akdong486@konkuk.ac.kr or kskonkuk@gmail.com; Tel. \& Fax.: +82-2-450-3742 

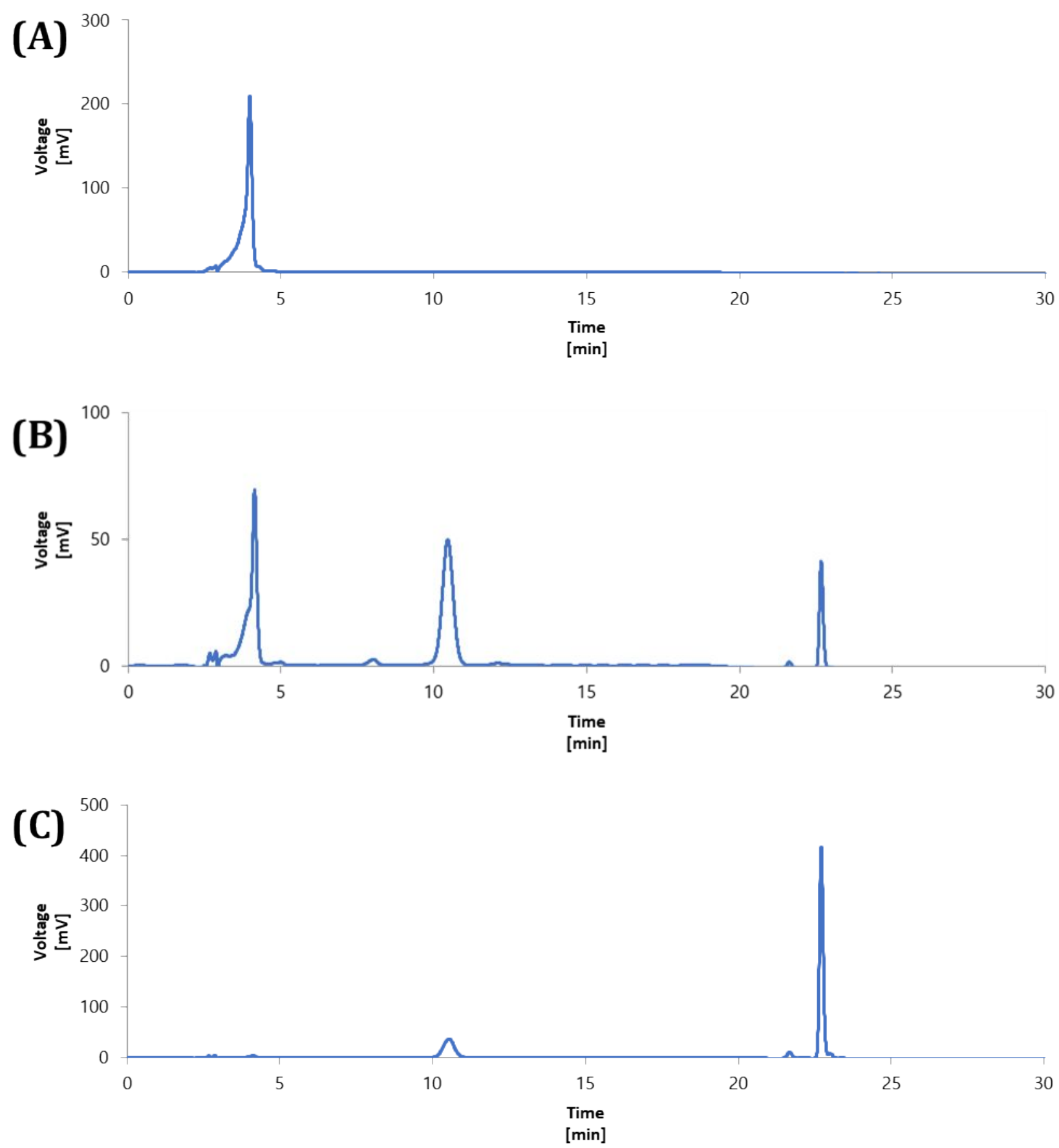

Figure S1. HPLC chromatograms of (A) synthetic Ast-N, (B) mixture of Ast-N, Ast-mE, and Ast-dE, and (C) Ast-dE. The synthetic Ast-N (A) was purchased from Sigma-Aldrich (SML0982), and the samples (B and C) were respectively prepared by esterification of synthetic Ast-N with the decanoic acids at different amounts. Specifically, the sample (B) was prepared with 6 times higher amount of decanoic acids than that of sample (C). 


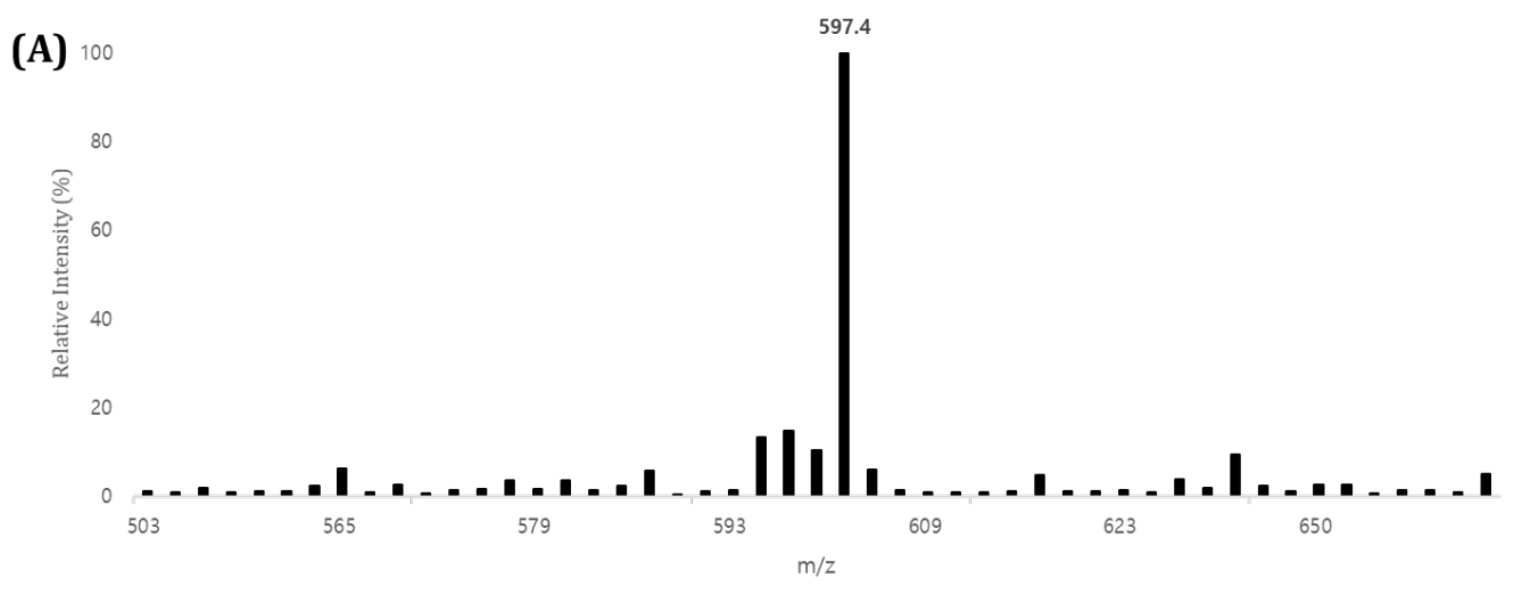

(B)

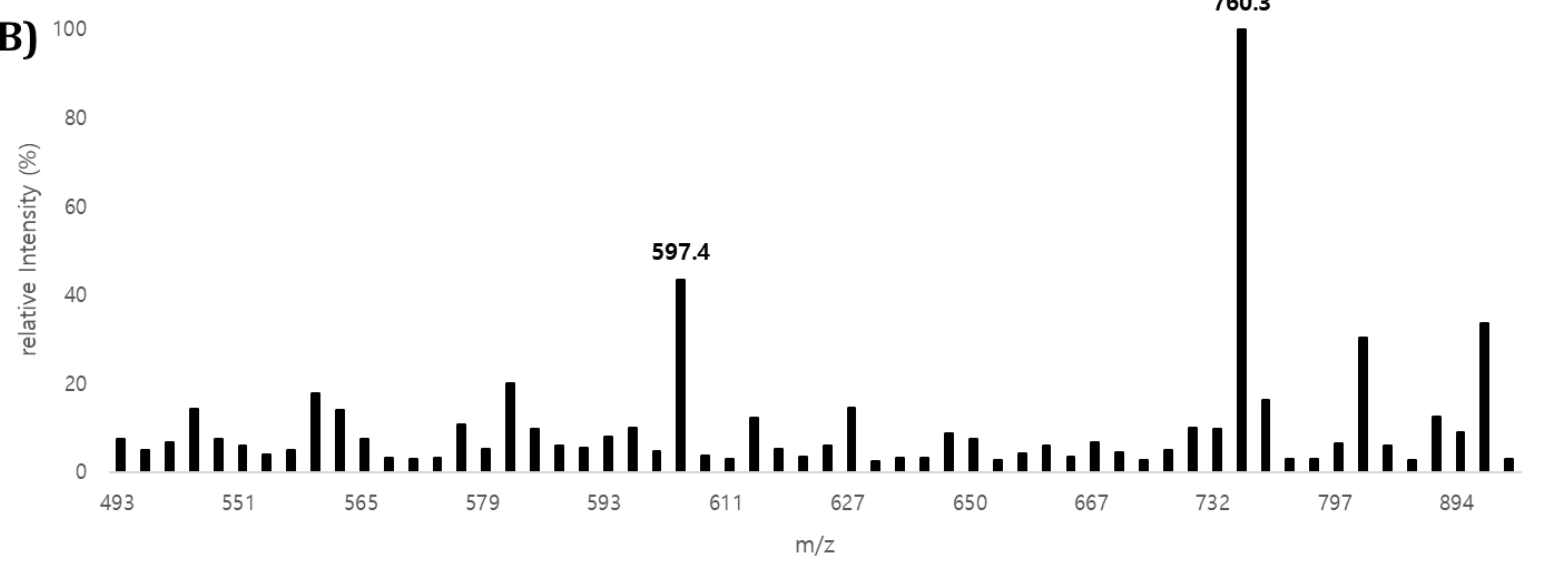

Figure S2. Mass spectra of (A) Ast-N in sample 2 and (B) Ast-N and Ast-mE in sample 3. LC-MS was performed on a Waters 2996 modular HPLC system coupled to a Q-TOF Ultima (UK) mass spectrometer. Mass spectra were acquired with a m/z 300-1,500 scan range. the significant ions were only found in a m/z 500 and 1,000 scan range. The $\mathrm{m} / \mathrm{z}$ peak at 597.4 was designated as Ast- $\mathrm{N}$ in sample 2 and $\mathrm{m} / \mathrm{z}$ peak at 597.4 and 760.3 are assigned as Ast-N and Ast-mE with $\mathrm{C} 10$ in sample 3, respectively. 


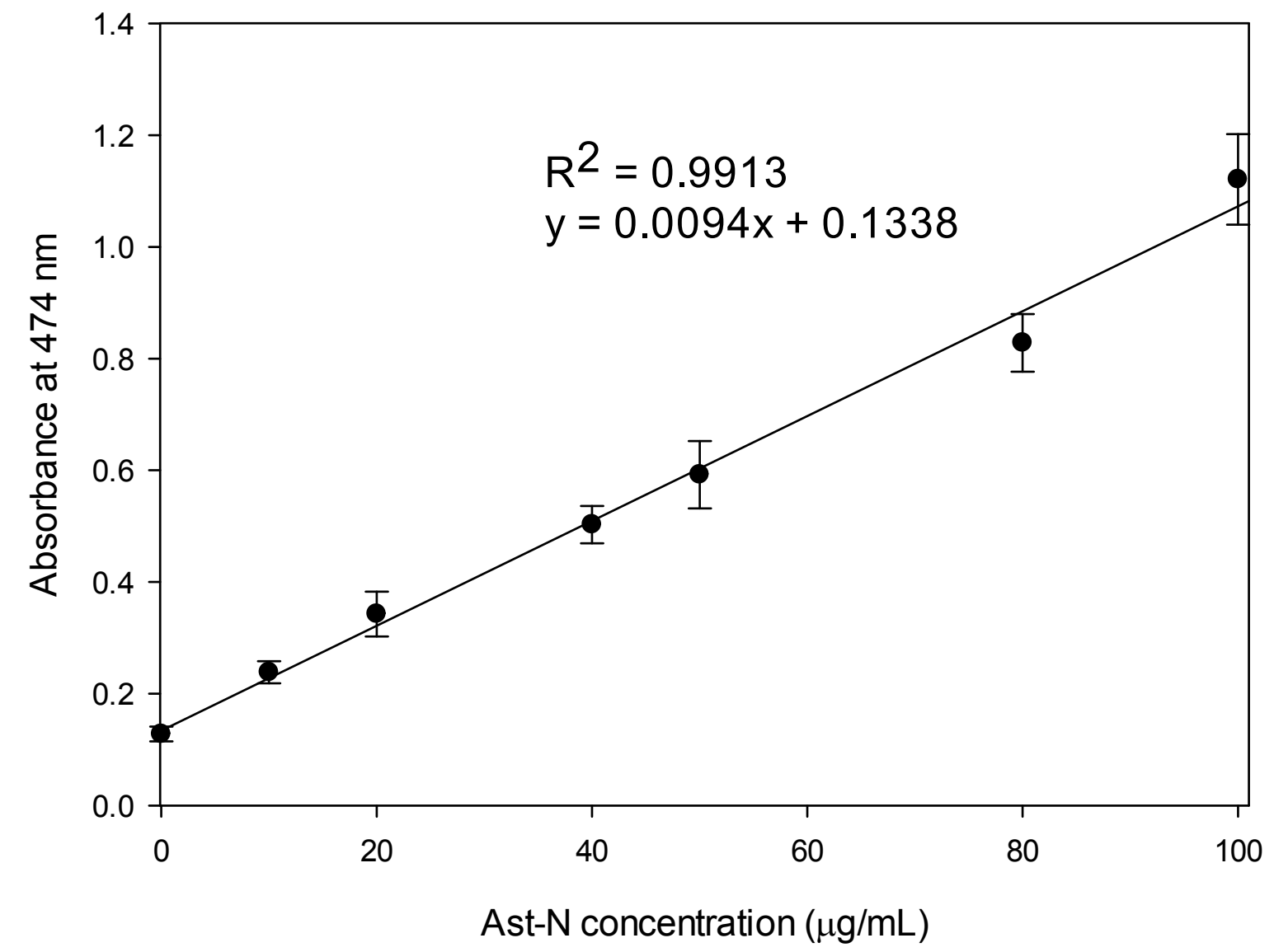

Figure S3. Calibration curve obtained by measuring the absorbance of different concentrations of Ast- $\mathrm{N}$ as the analytical standard, at $474 \mathrm{~nm}$. The results are presented as the mean \pm SD of three independent experiments. 


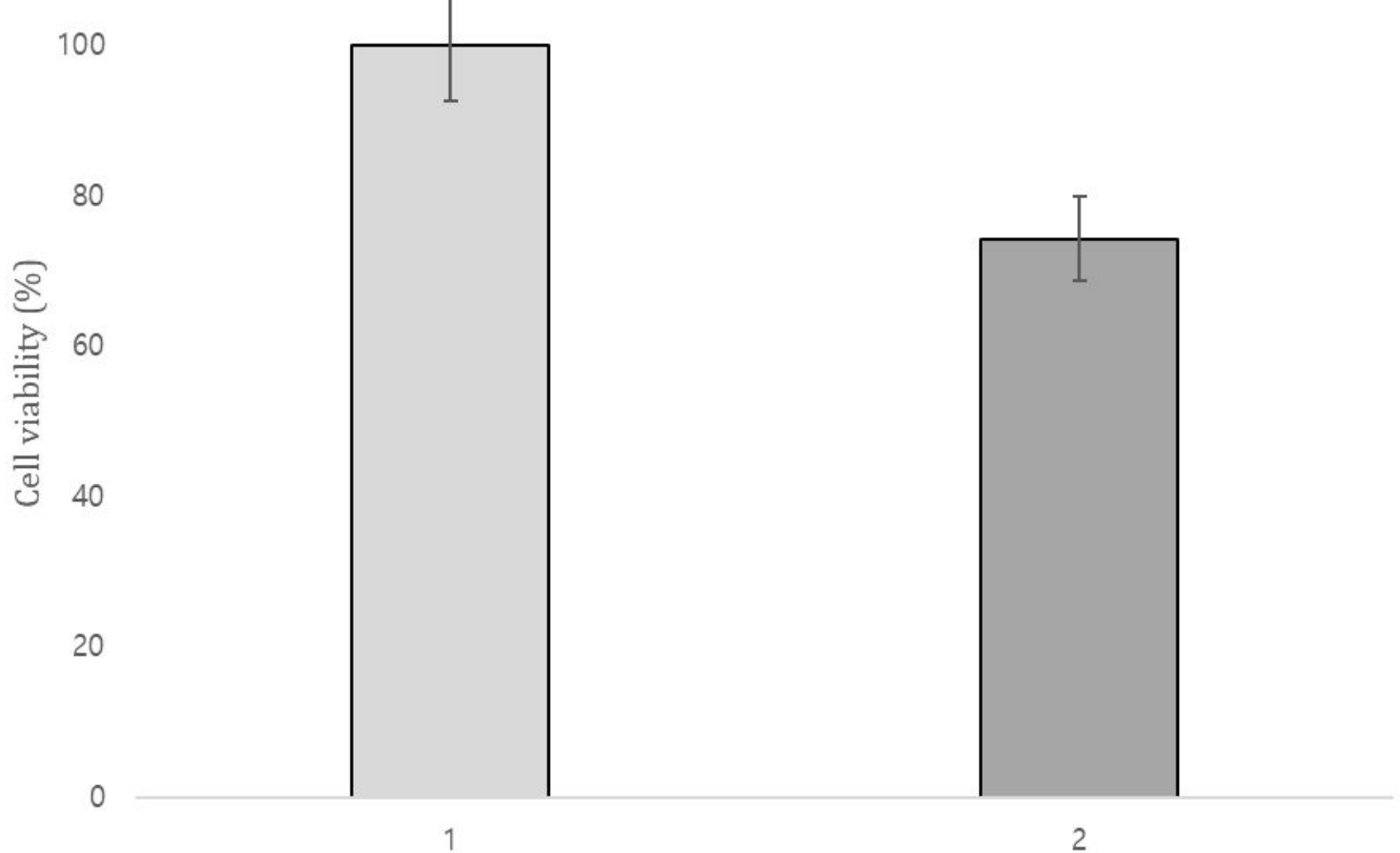

Figure S4. Viability of Raw 264.7 cells exposed to acetone (5\%), which was calculated through MTT assay. 1: without acetone, 2: with acetone (5\%). The bars represent the standard deviation obtained in three independent experiments. 